\title{
L'innovation en PME et son accompagnement par les TIC : quels effets sur la performance?
}

\author{
François DELTOUR*, Virginie LETHIAIS** \\ * Ecole des Mines de Nantes, LEMNA \\ ** Telecom Bretagne, ICI, Marsouin
}

\section{Résumé :}

La capacité à innover des entreprises ainsi que leurs ressources TIC sont généralement reconnues comme des gages de leur compétitivité. Dans notre article, ces deux sources de performance sont étudiées dans le contexte des Petites et Moyennes Entreprises (PME), en analysant notamment les retombées de l'accompagnement de l'innovation par les TIC. Notre recherche s'appuie sur une double investigation : une enquête menée auprès de PME de la région Bretagne et une collecte en ligne des performances financières de ces mêmes entreprises. Sur un échantillon final de 1086 entreprises, nous évaluons économétriquement dans quelle mesure la performance des PME peut être expliquée par l'innovativité, les ressources TIC (humaines et matérielles), ainsi que par l'effet combiné de l'innovativité accompagnée par les technologies de l'information. Le recours à une variable d'interaction mesurant les TIC directement mobilisées pour soutenir l'innovation des PME constitue l'apport méthodologique de notre recherche. L'investigation montre que les technologies de l'information contribuent à augmenter la performance des PME lorsqu'elles accompagnent des innovations : l'innovativité des PME a des retombées positives sur leur performance dans la mesure où elle est accompagnée par des investissements en TIC ou bien par un usage plus poussé des technologies déjà présentes dans l'entreprise. A contrario, l'innovativité et les ressources TIC considérées isolément minorent le niveau de performance des firmes étudiées. Ces différents résultats sont discutés au regard des caractéristiques spécifiques des PME.

\section{Mot-clés :}

Innovativité, technologies de l'information (TIC), Petites et Moyennes Entreprises, performance

Ceci est la version finale du manuscrit accepté pour publication. Des éléments de présentation ou de détails typographiques peuvent différer de la version publiée. 


\title{
Innovation in SMEs and ICTs' support: An assessment of their performance impacts
}

\author{
François DELTOUR*, Virginie LETHIAIS** \\ * Ecole des Mines de Nantes, LEMNA \\ ** Telecom Bretagne, ICI, Marsouin
}

\begin{abstract}
:
Firms' innovativeness and their ICT resources are seen as two factors of firms' competitiveness. Our research tests these sources of performance in the context of small-andmedium enterprises (SMEs) and examines possible synergies with ICTs supporting innovation. Our research is based on a double empirical investigation: a survey is conducted among firms in the region of Brittany (France), complemented with an online investigation of their financial performance. With a final sample of 1086 firms, we perform an econometric assessment of SMEs performance, explained by their innovativeness, their ICT resources (skills and assets) and the combined effect of their innovativeness supported by ICTs. The interaction variable capturing the combined effect constitutes the major methodological originality of our research. Our investigation shows that ICTs contribute to performance improvement when supporting innovations: SMEs' innovativeness positively influences performance when it is accompanied by specific investments in ICTs or by more intensive use of existing ICTs in the firm. However, innovativeness and the level of ICT resources have a direct negative effect on SMEs' financial performance. We discuss these results in the specific SMEs organizational context.
\end{abstract}

\section{Keywords:}

Innovativeness, information technologies (ICT), Small and Medium Enterprises, performance

This is an electronic reprint of the original article. This reprint may differ from the original in pagination and typographic detail. 


\section{Introduction}

L'innovation est reconnue de longue date comme un facteur de bénéfices organisationnels, économiques et sociaux (Foray et Mairesse, 1999). Les entreprises sont incitées à innover afin de garantir leur compétitivité. Les Petites et Moyennes Entreprises (PME) n'échappent pas à cette injonction : de leur capacité à innover dépend leur avenir.

Analysée dans les recherches en sciences sociales, l'innovation est un concept large qui va audelà de la notion d'invention (Loilier et Tellier, 2013). Au niveau organisationnel, l'innovation peut se définir comme l'adoption d'un équipement, d'une politique, d'un programme, d'un processus, d'un produit ou d'un service qui est nouveau pour l'organisation qui l'adopte et qui peut être développé en interne ou bien acquis (Damanpour, 1991). La capacité d'une entité ou d'un individu à innover est généralement traduite par le terme d'innovativité ${ }^{1}$. L'innovativité organisationnelle peut être appréhendée comme la «capacité organisationnelle à innover, c'est-à-dire la propension d'une organisation ou sa volonté à réaliser des innovations » (Wang et Ahmed, 2004, p.303). Cette capacité se traduit au sein de l'organisation par un soutien et une perméabilité à l'innovation. L'entreprise se positionne comme "proactive dans l'exploration de nouvelles opportunités, plutôt que d'exploiter essentiellement ses forces actuelles » (Menguc et Auh, 2006, p.65).

Une étude récente du CIGREF (2013) intitulée «La contribution de l'IT à l'innovation » indique comment les Technologies de l'Information et de la Communication (TIC) peuvent être intégrées à chacune des étapes de la démarche d'innovation dans les entreprises, et donc influencer positivement leurs retombées. Appréhender les TIC comme levier des bénéfices retirés de l'innovativité des entreprises constitue la préoccupation centrale de notre recherche. Concrètement, ce rôle des TIC comme levier provient de leur capacité à accélérer le processus d'innovation ou à favoriser la capitalisation des savoirs R\&D de l'entreprise (Pavlou et El Sawy, 2006) ou bien encore de leur capacité à mieux identifier les besoins émergents du marché (Tambe et al., 2012). Plusieurs travaux ont cherché à expliquer comment la propension des entreprises à associer leur capacité d'innovation à leur usage des technologies de l'information pouvait affecter leur performance (Huang et Liu, 2005 ; Dibrell et al., 2008 ; Kmieciak et al. 2012 ; Raymond et al., 2013). Notre recherche s'inscrit dans le prolongement de ces travaux, en étudiant le cas des Petites et Moyennes Entreprises, c'est-à-dire le cas d'un fonctionnement organisationnel spécifique (Marchesnay, 1991). La PME est en effet généralement vue comme un contexte organisationnel offrant une certaine réceptivité à l'innovation, même si celle-ci n'est pas toujours couronnée de succès (Oseo, 2011). Parallèlement, la capacité de la PME à mettre à profit les TIC est souvent questionnée (Poulin et Tran, 2010). Notre question de recherche est alors formulée de la manière suivante : dans quelle mesure la combinaison de leur stratégie d'innovation et de leur politique TIC est-elle bénéfique aux PME? Plus précisément, nous cherchons à évaluer dans quelle mesure la performance des PME peut être expliquée par l'innovativité, les ressources TIC (humaines et matérielles), ainsi que l'effet combiné de l'innovativité accompagnée par les technologies de l'information.

Pour cela, nous mobilisons les résultats d'une enquête menée auprès de PME de la région Bretagne. Un échantillon représentatif de 1992 PME a renseigné un questionnaire portant sur leur politique en matière d'équipement et d'usage des TIC, ainsi que leurs pratiques d'innovation. Cette enquête a été complétée par une investigation sur les performances financières des PME concernées. Les traitements statistiques et économétriques sont réalisés sur un échantillon final de 1086 entreprises.

\footnotetext{
${ }^{1}$ Le terme anglo-saxon « innovativeness » peut être traduit en français par innovativité. Il est également traduit par d'autres expressions comme « tendance à innover» ou « capacité à innover».
} 
Notre article présente dans un premier temps les concepts mobilisés ainsi que l'approche combinée retenue dans notre recherche (section 1). La deuxième partie détaille l'investigation quantitative qui a été menée (section 2). Enfin, les résultats obtenus sont présentés et discutés (section 3).

\section{La performance des PME expliquée par leur innovativité et leurs ressources en TIC}

Les notions d'innovativité et d'innovation sont assez proches et ont été utilisées de façon parfois indistincte (Damanpour, 1991). L'innovativité, en tant que capacité à innover, s'applique à une entité, c'est-à-dire un individu, une organisation ou même une économie. Au niveau organisationnel, l'innovativité est parfois réduite à la capacité des entreprises à générer des innovations de produits. Or, l'innovation concerne aussi la capacité à mettre en œuvre toutes les activités en amont, notamment les innovations de procédés (Subramanian, 1996). Il s'agit donc d'une capacité à mettre en place à l'intérieur de l'organisation toute nouveauté, que cette nouveauté existe ou non ailleurs.

\section{I.1. Les effets directs de l'innovativité et des TIC}

\section{I.1.1. La contribution de l'innovativité à la performance des PME}

Les efforts réalisés par une organisation pour innover peuvent se comprendre comme un investissement visant à générer, à termes, des retombées positives (croissance, profits, etc.). Néanmoins, toute nouveauté induit une prise de risque et ne signifie pas le succès de l'innovation, qu'il s'agisse d'un succès commercial ou de gains d'efficience ; cela questionne le lien à la performance. Dans l'abondante littérature sur le management de l'innovation, le lien entre innovativité et performance est souvent appréhendé de manière positive (Foray et Mairesse, 1999; Loilier et Tellier, 2013). La contribution positive de l'innovativité à la performance des entreprises est démontré dans de nombreux travaux (Subramanian, 1996; Hult et al., 2004 ; Das et Joshi, 2012). Cependant, certaines recherches tempèrent cette idée, en ne validant pas de relation directe (Jansen et al., 2006 ; Dibrell et al., 2008). Pour d'autres auteurs, l'innovativité et la performance sont reliés de façon curvilinéaire (Huang et Liu, 2005) : seule une politique d'innovation modérée, ni trop forte ni trop faible, serait bénéfique à l'entreprise.

Le contexte organisationnel des PME permet de préciser le lien de l'innovativité à la performance. En effet, les PME ne peuvent pas uniquement être considérées comme une version réduite des grandes entreprises, notamment lorsqu'il s'agit de petites entreprises familiales : leur mode de fonctionnement diffère sur de nombreux points des grandes firmes. C'est en particulier le cas concernant les ressources financières ou humaines à disposition. Il peut donc exister des différences entre les résultats obtenus sur de grandes entreprises et sur des PME. Plusieurs arguments contradictoires concernant la capacité à innover des PME et leurs retombées peuvent être avancés (Hausman, 2005) : certaines particularités, comme par exemple la capacité à répondre aux changements de l'environnement, se traduisent par un niveau élevé de flexibilité organisationnelle et d'innovation. La proximité avec les clients ou les partenaires externes peut également favoriser le déploiement réussi d'innovations. Inversement, l'absence au sein des PME de compétences internes peut être un frein à l'innovation ou à son succès sur le marché. De plus, la sur-implication personnelle et 
opérationnelle du dirigeant, sa personnalité, ou bien les considérations familiales peuvent être un frein à la prise de risques. Via des études de cas en PME, Hadjimanolis (2000) confirme le rôle de certains facteurs pour expliquer l'innovativité de ces firmes, comme les caractéristiques des propriétaires/dirigeants. En définitive, même si elle est menée dans un contexte organisationnel spécifique, l'innovativité en PME apparaît comme une source potentielle de bénéfices.

\section{I.1.2. La contribution des TIC à la performance des PME}

Les retombées des technologies et des systèmes d'information ont longtemps été sujettes à caution. Ce phénomène a été popularisé par le paradoxe de productivité des TIC énoncé par Solow. De nombreux travaux, souvent de nature économétrique, ont permis d'obtenir des résultats plus probants, mais pas toujours généralisables, notamment du fait d'approches méthodologiques trop divergentes (Aral et Weill, 2007). De même, la nature des technologies considérées évolue au fur et à mesure des années, amenant des causalités antérieurement validées à ne plus être vérifiées du fait d'une disponibilité accrue et d'un coût réduit des technologies les plus récentes (Chae et al., 2014). Malgré ces réserves, la majorité des recherches empiriques disponibles indique que l'effet des TIC sur la productivité est positif et significatif (Cardona et al., 2013).

La contribution des TIC à la performance peut s'analyser comme le développement d'une capacité spécifique à la firme qui lui offre la possibilité de développer un avantage concurrentiel à l'égard de ses concurrents (Liang et al. 2010). La revue de littérature proposée par Melville et al. (2004) tend ainsi à montrer que les ressources TIC génèrent de la valeur au niveau des organisations, sachant que cette valeur dépendra également du niveau de ressources complémentaires, du climat concurrentiel ainsi que de la situation macroéconomique.

Qu'en est-il pour les PME ? L'étude du rôle et des spécificités des systèmes d'information dans le fonctionnement des petites entreprises a fait l'objet d'un certain nombre de contributions (Premkumar, 2003 ; Poulin et Tran, 2010) montrant que la relation à l'informatique n'est pas évidente pour les organisations de petites tailles. Les PME développent très souvent un « sentiment d'impuissance face aux TIC » (Monnoyer et Boutary, 2008, p.106). Allant aux delà de ces possibles difficultés, Rougès et al. (2010) passent en revue les travaux ayant investigué spécifiquement le lien avec la performance des PME. Parmi la vingtaine de travaux quantitatifs identifiés, le lien des TIC à la performance est très fréquent, même s'il n'est pas systématique. Ce lien est parfois influencé par certains facteurs, notamment la manière dont s'est déroulée l'implantation des TIC concernées, leur complexité, leur nombre ou les compétences des personnes associées. Les compétences informatiques internes pouvant faire défaut aux PME (Poulin et Tran, 2010), nous mettons en avant cette dimension, de manière complémentaire à celle des actifs TIC détenus et utilisés dans les PME. Il est alors possible de considérer deux dimensions se combinant pour former les ressources en TIC (Melville et al., 2004 ; Aral et Weill, 2007) : (1) les «actifs TIC» qui correspondent aux différents équipements et investissements et (2) les «compétences en TIC » qui correspondent aux différentes compétences internes relatives à l'informatique, combinées aux pratiques autour des TIC développées dans l'organisation. La capacité en TIC qui en découle repose sur la combinaison de ces ressources matérielles et humaines (Bharadwaj, 2000). En définitive, les ressources TIC - aussi bien humaines que matérielles sont appréciées comme un facteur pouvant contribuer à la performance des PME. 


\section{I.2. Le soutien des TIC à l'innovativité}

\section{I.2.1. Les effets combinés entre TIC et innovation : des résultats contradictoires}

Les technologies de l'information ont-elles une influence sur la relation entre innovativité et performance des PME? A cette question centrale dans notre recherche, les investigations antérieures - toutes quantitatives - ont donné des résultats se révélant contrastés. En effet, parmi les quatre travaux identifiés sur ce thème, deux concluent à un rôle positif d'accompagnement par les TIC, alors que deux autres ne trouvent pas d'effet combiné, voire un effet négatif.

Les travaux de Huang et Liu (2005) puis Dibrell et al. (2008) concluent à un effet positif. Huang et Liu (2005), sur la base d'une investigation auprès de 297 grandes entreprises taïwanaises, montrent que la variable d'interaction entre le capital innovation des entreprises (taux de R\&D) et le capital en TIC (taux de dépenses TIC) est positivement liée à la performance, montrant un effet de synergie. En revanche, ils concluent que le capital en TIC n'influence pas de façon directe la performance de la firme et que le capital innovation a un impact direct non linéaire sur la performance (positif jusqu'à un certain seuil et négatif audelà). Dibrell et al. (2008), s'ils concluent à une absence de lien direct entre innovation et performance, mettent en évidence une relation indirecte via l'importance donnée aux TIC par les dirigeants. Leur modèle, testé par questionnaire auprès de $311 \mathrm{PME}$ étasuniennes, met donc en avant le rôle médiateur des TIC sur la performance.

Les travaux récents de Kmieciak et al. (2012) et Raymond et al. (2013) sont plus réservés quant à la contribution des TIC. L'étude de Kmieciak et al. (2012), par régression multiple sur les données de 109 PME polonaises, ne confirme pas le fait que la capacité en TIC modère la relation entre l'innovativité et la performance de la firme. Pour leur part, Raymond et al. (2013) réalisent une investigation auprès de 309 PME manufacturières canadiennes et montrent qu'il y a bien un effet de la capacité à innover (investissements en R\&D) sur la croissance et la productivité de ces firmes. Cependant, le recours accru par ces entreprises à des TIC dédiées à l'intégration des processus (ERP, MRP, EDI...) ne renforce pas l'effet sur leur performance : l'effet combiné sur la croissance n'est pas statistiquement significatif, alors que l'effet combiné sur la productivité est lui significativement négatif. Les auteurs parlent alors d'un effet paradoxal de l'intégration des TI : les firmes possédant des TIC intégratives perdent dans une certaine mesure les bénéfices des nouveaux produits ou des nouveaux procédés car ceux-ci entreraient en conflit avec les processus existants, inscrits dans les TIC dites intégratives. La modification des processus existants entraînerait des coûts en temps ainsi que de nombreux problèmes techniques et humains à résoudre, affectant la productivité de l'organisation.

Ces différents résultats, non concordants, nous amènent à clarifier la question du rôle des TIC comme levier - ou non - des effets de l'innovation sur la performance. Une piste de clarification est que les travaux cités analysent et mesurent de façon juxtaposée les pratiques d'innovation et les politiques en TIC des entreprises, sans savoir si le lien entre TIC et innovativité est effectif dans les entreprises. Ils ne mesurent donc qu'indirectement la manière dont l'accompagnement du processus d'innovation par les TIC peut se réaliser.

\section{I.2.2. Les bénéfices de l'accompagnement de l'innovation par les TIC}

Les ressources TIC étant diffuses dans de nombreuses activités et dans de nombreux processus des entreprises, leurs effets potentiels sont multiples. Afin de clarifier ces effets, il 
est possible de se focaliser sur une activité spécifique, comme celle d'innovation. Différentes TIC peuvent renforcer la capacité d'innovation des entreprises ou en renforcer les retombées. Dans le contexte de l'innovation produit, Pavlou et El Sawy (2006) identifient trois situations dans lesquelles les compétences de développement de nouveaux produits peuvent être renforcées par les TIC : l'usage des systèmes de gestion de projet et gestion des ressources, l'usage des systèmes de gestion de connaissance, l'usage des systèmes de travail collaboratif. Concernant cette dernière activité, Banker et al. (2006) montrent que l'utilisation des outils et logiciels collaboratifs renforce effectivement la collaboration entre les personnes chargées de l'innovation. En conséquence, cette utilisation réduit le cycle de développement, réduit les coûts de développement, accroit les possibilités de réalisations différentes et les réutilisations, et enfin améliore la qualité du produit conçu. De la même manière, Merminod et al. (2009) montrent comment les systèmes les plus récents comme le PLM (Product Life Management) participent à accroître conjointement la productivité et la fiabilité du développement de nouveaux produits. Tambe et al. (2012) analysent comment les pratiques de collecte externe d'information soutenues par les TIC sont source d'innovation (de produit) et de productivité. Parallèlement, Kmieciak et al. (2012) avancent l'idée que certaines catégories de TIC permettent une meilleure compréhension du marché en facilitant les échanges avec les clients, qui participent ainsi (via les e-mails, les forums de discussion, les réseaux sociaux) à concevoir des produits répondant à leurs attentes. Même si cette ouverture aux clients reste souvent limitée (Kuusisto et Riepula, 2011), elle peut être dans certains cas très forte (comme par exemple les pratiques de crowdsourcing dédiées à l'innovation ou des pratiques d'open innovation). Enfin, les technologies de l'information sont largement reconnues dans la littérature pour leur contribution à l'innovation de processus, car porteuses de transformation de l'organisation (Besson et Rowe, 2011).

En définitive, les travaux cités s'accordent sur l'idée que la mobilisation des technologies de l'information est source de gains pour l'innovation : les TIC améliorent la manière dont les entreprises innovent; et cela, dans une diversité de situations d'innovation (développement de nouveaux produits, adoption de nouveaux procédés, etc.) et en mobilisant une large palette de technologies (qu'elles soient génériques ou dédiées à l'innovation). Nous poursuivons le raisonnement ainsi mené, en faisant l'hypothèse que cet accompagnement de l'innovation par les TIC constitue un levier de performance qui bénéficie à la firme dans son ensemble.

\section{Une investigation empirique auprès de PME}

La méthode retenue dans notre recherche est une investigation quantitative via une double collecte de données. Un questionnaire adressé aux PME de la région Bretagne a permis le recueil de l'essentiel des variables utilisées dans la recherche. La performance - appréhendée via des données financières - a fait l'objet d'un recueil spécifique.

\section{II.1. L'enquête régionale auprès de PME}

L'enquête, menée par le Groupement d'Intérêt Scientifique (GIS) M@rsouin en collaboration avec la CCI de Bretagne, porte sur l'utilisation des technologies de l'information par les PME de la région (Marsouin, 2009). La population ciblée par cette enquête est les entreprises de 10 à 250 salariés appartenant aux secteurs de l'industrie, du commerce et des services (à l'exception de l'agriculture et des services publics). L'enquête repose sur un questionnaire général, qui interroge d'abord chaque PME sur son activité et sa situation générale, et énumère ensuite une large palette d'équipements et leur utilisation possible à l'intérieur de la firme, ou bien avec des partenaires extérieurs. Une série de questions porte sur la politique 
d'innovation de l'entreprise, ainsi que son accompagnement par les TIC. Nous précisons cidessous la manière dont ont été appréhendées les mesures mobilisées dans notre recherche. Les questions correspondantes issues de l'enquête sont disponibles en Annexe.

\section{II.1.1. Mesure de l'innovativité et son soutien par les TIC}

\section{L'innovativité}

Plusieurs approches existent pour mesurer l'innovativité. La capacité à innover peut être mesurée via le niveau des investissements en R\&D (Huang et Liu 2005 ; Raymond et al., 2013) ou au travers du dépôt de brevets (Kleis et al., 2012). Même si ces mesures sont parfois utilisées dans le contexte d'investigations en PME, elles se révèlent peu adaptées aux plus petites de ces entreprises qui formalisent peu leurs moyens d'innovation alors qu'elles peuvent néanmoins développer une réelle capacité à mettre en place des innovations (Forsman, 2011). Une approche alternative consiste alors à estimer la capacité que l'entreprise a pu avoir à mettre en œuvre différentes formes d'innovation dans un temps récent. Ainsi, Subramanian (1996) insiste sur le fait que la mesure de l'innovativité doit être faite dans la durée et également de façon multidimensionnelle. Parmi les différentes dimensions de l'innovativité (Wang et Ahmed, 2005), la plus classique porte sur la distinction entre la capacité à innover en produit et celle en procédé (Dibrell et al., 2008 ; Forsman, 2011 ; Raymond et al., 2013). Les innovations de produit portent sur un changement des produits ou des services fournis au client alors que les innovations de procédé concernent la manière dont une entreprise fabrique ses produits. Suivant cette catégorisation, les entreprises sont interrogées sur ces deux types d'innovations. Enfin, nous suivons les recommandations de Subramanian (1996) qui souligne l'importance d'indiquer un laps de temps dans lequel l'innovation a pu se réaliser. L'innovativité est donc mesurée par la capacité à avoir innové, en produit ou en procédé, dans les 24 derniers mois avant l'enquête. La variable innovativité prend donc la valeur 1 si l'entreprise s'est positionnée sur de nouveaux produits ou services ou a introduit de nouveaux procédés dans les deux dernières années, et 0 sinon.

\section{Le soutien des TIC à l'innovation}

Une originalité forte de l'enquête réalisée est qu'elle investigue de manière directe dans quelle mesure l'innovativité est accompagnée par les technologies de l'information. Les différents travaux analysant la manière dont les technologies de l'information sont impliquées dans le processus d'innovation montrent qu'il existe une grande diversité d'applicatifs possibles, que ceux-ci soient dédiés (comme les applicatifs de gestion de projet ou le PLM) ou qu'ils soient plus généraux (comme les e-mails ou les systèmes de gestion des connaissances). En conséquence, il est difficile d'appréhender les contours des TIC pour l'innovation. Cette appréhension semble d'autant plus difficile dans le contexte des PME, où les situations d'innovation rencontrées peuvent être très disparates : il existe un fossé entre la PME qui a peu de moyens financiers pour investir dans une technologie dédiée et celle dont l'innovation est au cœur du modèle d'affaires. L'activité de mise en place de nouveaux produits ou nouveaux procédés peut en effet s'appuyer sur des investissements spécifiques en TIC, et elle peut également s'appuyer sur les TIC déjà présentes dans l'entreprise qui seront mobilisées de façon plus intense. Plutôt que de formuler des hypothèses sur les types de technologies susceptibles d'accompagner les innovations des entreprises de notre échantillon, nous avons demandé aux entreprises si les innovations réalisées dans les 24 derniers mois ont donné lieu, soit à des investissements spécifiques en TIC, soit à un usage accru des TIC à disposition, sans distinguer spécifiquement les technologies concernées. Les deux items d'investissement et d'usage sont agrégés dans la suite de l'analyse. 


\section{II.1.2. Mesure des ressources TIC}

Les ressources TIC de la PME sont caractérisées selon deux dimensions complémentaires, notamment mises en avant par Aral et Weill (2007) : les compétences informatiques internes et les actifs TIC.

\section{Les compétences informatiques internes}

Les compétences informatiques internes correspondent à l'investissement en ressources humaines dédiées à l'informatique de l'entreprise. La variable compétences informatiques peut prendre quatre modalités : 3 si l'entreprise dispose d'un service informatique constitué, 2 si elle dispose d'au moins un salarié à mi-temps ou plus dédié à l'informatique (mais pas de service informatique constitué), 1 si elle dispose d'au moins un salarié diplômé du supérieur en informatique (mais pas de salarié spécifiquement dédié à l'informatique et pas de service informatique constitué) et 0 si elle ne dispose d'aucune de ces ressources en interne.

\section{Les actifs TIC mesurés selon différents niveaux de granularité}

Les actifs TIC correspondent à l'investissement en équipements et logiciels dans l'entreprise. Dans notre investigation, plusieurs approches sont retenues, adoptant un niveau de granularité plus ou moins fin des actifs TIC présents dans chaque entreprise. Quatre mesures sont utilisées :

- Investissement agrégé en TIC : afin d'apprécier le niveau global des investissements en actifs TIC, une évaluation comparative permet au répondant de positionner son entreprise par rapport à ses principaux concurrents ou à son secteur (Dibrell et al., 2008). Dans notre recherche, l'évaluation des investissements TIC est mesurée par un positionnement de l'entreprise au regard de son secteur d'activité, les investissements en TIC pouvant être supérieurs, inférieurs ou bien dans la moyenne du secteur.

- Informatisation des fonctions : pour un large éventail de fonctions possibles au sein d'une entreprise, le questionnaire nous indique si chacune est assurée en interne, externalisée ou inexistante. Le taux d'informatisation des fonctions est une variable continue entre 0 et 1 qui représente le nombre de fonctions informatisées dans l'entreprise rapporté au nombre de fonctions assurées en interne.

- Adoption des applicatifs: nous retenons un ensemble de douze équipements ou applicatifs parmi les plus représentatifs des usages en entreprise : site web (marchand ou non marchand), workflow, agendas partagés, visioconférence, ERP, etc. La liste complète est présentée dans le schéma 4 et en annexe. Pour chaque PME, un score d'adoption des applicatifs est établi et compris entre 0 et 12 .

- Adoption de catégories d'applicatifs : les douze équipements et applicatifs retenus sont regroupés par catégories, selon la logique des classifications existantes (Aral et Weill, 2007 ; Raymond et Bergeron, 2008). Adaptée de ces classifications, notre approche distingue les applicatifs d'automatisation (logiciels "métier" spécifiques, logiciels de workflow, ERP), les applicatifs d'information et de communication (site web non marchand, listes de diffusion, téléphonie sur IP), les applicatifs de collaboration (espaces de travail partagés, agendas partagés, visioconférence) et enfin, les applicatifs marchands (site web marchand, EDI, certificats électroniques). Pour chaque catégorie d'applicatif, la variable de niveau d'adoption est donc comprise entre 0 et 3 . 


\section{II.1.3. Mesures complémentaires}

\section{Le dynamisme du marché}

Le dynamisme du marché est reconnu comme pouvant affecter les performances des entreprises. Ainsi, l'étude de Jansen et al. (2006) révèle que le dynamisme de l'environnement ainsi que le degré de concurrence participent sous certaines conditions à la performance financière des unités innovatrices. Dans notre investigation, le dynamisme du marché prend trois modalités qui correspondent à un marché principal déclaré par la PME comme étant en croissance, stable ou en décroissance.

\section{Le secteur d'activité}

Les caractéristiques spécifiques des différents secteurs d'activités peuvent se traduire par des écarts en termes de performance des firmes. A partir des codes APE des entreprises interrogées, douze secteurs d'activité sont retenus dans notre analyse (la liste détaillée est disponible en Annexe).

\section{II.2. Mesure de la performance des PME}

Appréhender la performance globale d'une entreprise n'est jamais simple. Les travaux évaluant la performance induite par les TIC ont retenu des approches multiples opposant régulièrement les mesures perceptuelles à des mesures objectives, ces dernières étant plus difficiles à obtenir (Tallon et Kraemer, 2007). Les mesures objectives sont d'ailleurs largement minoritaires parmi les travaux mesurant la performance des PME (Rougès et al., 2010). Le choix a été fait d'opter pour une mesure objective afin de minimiser les éventuels biais dans l'appréciation de la performance. En ligne avec les recherches quantitatives antérieures (Bharadwaj, 2000; Huang et Liu, 2005; Aral et Weill, 2007), une mesure financière par ratio est adoptée. Nous retenons le taux de marge d'exploitation qui est le résultat d'exploitation de l'entreprise rapporté à son chiffre d'affaires et multiplié par 100 (RE/CA x 100). Ce ratio permet de prendre en compte le profit opérationnel de la PME en le rapportant à sa taille, ici mesurée par le niveau des ventes.

La mesure de la performance est réalisée avec un décalage temporel de deux années, postérieurement à la mesure des activités d'innovativité et d'investissement TIC. Ce laps de temps reflète le délai nécessaire à l'obtention d'éventuelles retombées. L'estimation de ce laps est une question difficile qui n'a pas donné de réponse définitive dans l'étude des retombées des TIC (Kohli et Devaraj, 2003 ; Cardona et al., 2013). De même, le laps de temps entre l'innovation (dépenses R\&D, brevets, nouveaux produits ou procédés) et ses impacts sur la performance de l'entreprise est variable. Ainsi, Kafouros et Wang (2008) soulignent que le délai entre les dépenses $R \& D$ et la performance dépend notamment de la nature de l'innovation, de la taille de la firme et des opportunités technologiques ; ces dernières étant elles-mêmes très dépendantes du secteur d'activité. Les petites entreprises, peu enclines à financer des projets innovants de long terme, s'orienteront vers des projets susceptibles de générer des revenus à court terme (Kafouros, 2005). En choisissant un écart de deux années soit un laps de temps allant de deux à quatre ans pour l'innovativité de l'entreprise - nous choisissons un délai relativement court. Celui-ci nous semble bien adapté à la situation étudiée, car c'est un horizon temporel acceptable pour des PME qui pourraient difficilement s'engager sur des investissements d'innovation ayant des retombées sur un horizon plus lointain. 


\section{II.3. La collecte des données}

Le questionnaire a été envoyé à toutes les PME de la région Bretagne au printemps 2008, soit quelques 8000 destinataires recensés dans les registres de la CCI de la région Bretagne (Marsouin, 2009). Le questionnaire pouvait être rempli sur document papier et renvoyé par courrier, ou bien rempli directement en ligne. Afin d'augmenter le nombre de répondants, une administration complémentaire a été réalisée par téléphone. Les firmes ont été choisies afin d'obtenir une bonne représentativité finale des répondants en termes de localisation au sein des départements, de taille et de secteur d'activité, relativement au tissu économique régional (méthode des quotas). En définitive, ce sont 1992 réponses de PME qui sont collectées. Le répondant est, dans la majorité des cas, le dirigeant de l'entreprise ou le responsable administratif ou/et financier.

Partant de cette base de 1992 entreprises, le recueil des informations financières a été effectué via la retranscription systématique des informations disponibles sur des sites web spécialisés d'information financière, pour l'année 2010. Les informations suivantes ont ainsi pu être collectées pour un grand nombre d'entreprises : effectif, chiffre d'affaires, excédent brut d'exploitation et résultat d'exploitation. Les PME dont les données financières n'ont pas pu être obtenues sur les sites ont été écartées.

Plusieurs vérifications et filtrages successifs ont ensuite été réalisés afin d'ôter de l'analyse les PME pour lesquelles il pouvait y avoir une incohérence entre les informations déclarées et celles consultées en ligne :

- Un écart trop important entre l'effectif déclaré et l'effectif collecté en ligne (supérieur de $25 \%$ ) indiquait le risque que les données financières collectées correspondent à une autre entité que celle visée (par exemple, les données en ligne correspondent à celle du groupe dans son ensemble, et non pas uniquement l'entité du groupe ayant rempli le questionnaire) ;

- Les entreprises dont le chiffre d'affaires collecté via les sites web dépassait 50 millions d'euros ont également été écartées. En effet, ces sociétés ne font pas partie des PME mais des ETI (Entreprises de Taille Intermédiaire);

Enfin, les entreprises n'ayant pas répondu aux questions concernant l'innovativité et l'accompagnement des innovations par les TIC ont été exclues de l'analyse. En définitive, l'analyse empirique a pu être réalisée sur un échantillon de 1086 entreprises.

\section{Résultats et analyse}

\section{III.1. Résultats descriptifs de l'étude}

Les PME de l'échantillon ont un effectif moyen de 28 salariés (écart-type 26,6) et les principaux secteurs d'activités (dépassant les $10 \%$ de l'échantillon) sont - par ordre d'importance - la construction, les services aux entreprises, le commerce de détail, ainsi que l'industrie des biens intermédiaires et de l'énergie.

\section{III.1.1. L'innovativité modérée des PME}

Parmi les 1086 entreprises, 59\% n'ont pas innové dans les deux années précédant l'enquête (figure 1). Parmi les $41 \%$ qui ont innové, un plus grand nombre de PME se sont positionnées sur de nouveaux produits/services (35\%) que sur de nouveaux procédés (19\%). Ces derniers chiffres confirment le résultat d'Hausman (2005) obtenu par études de cas, selon lequel les 
PME sont plus enclines à adopter de nouveaux produits tangibles que de nouvelles idées ou pratiques de management. Les PME ayant innové en procédé ont également innové en produit pour un grand nombre d'entre-elles ; les entreprises ayant mené les deux formes d'innovation représentant $13 \%$ de l'échantillon total.

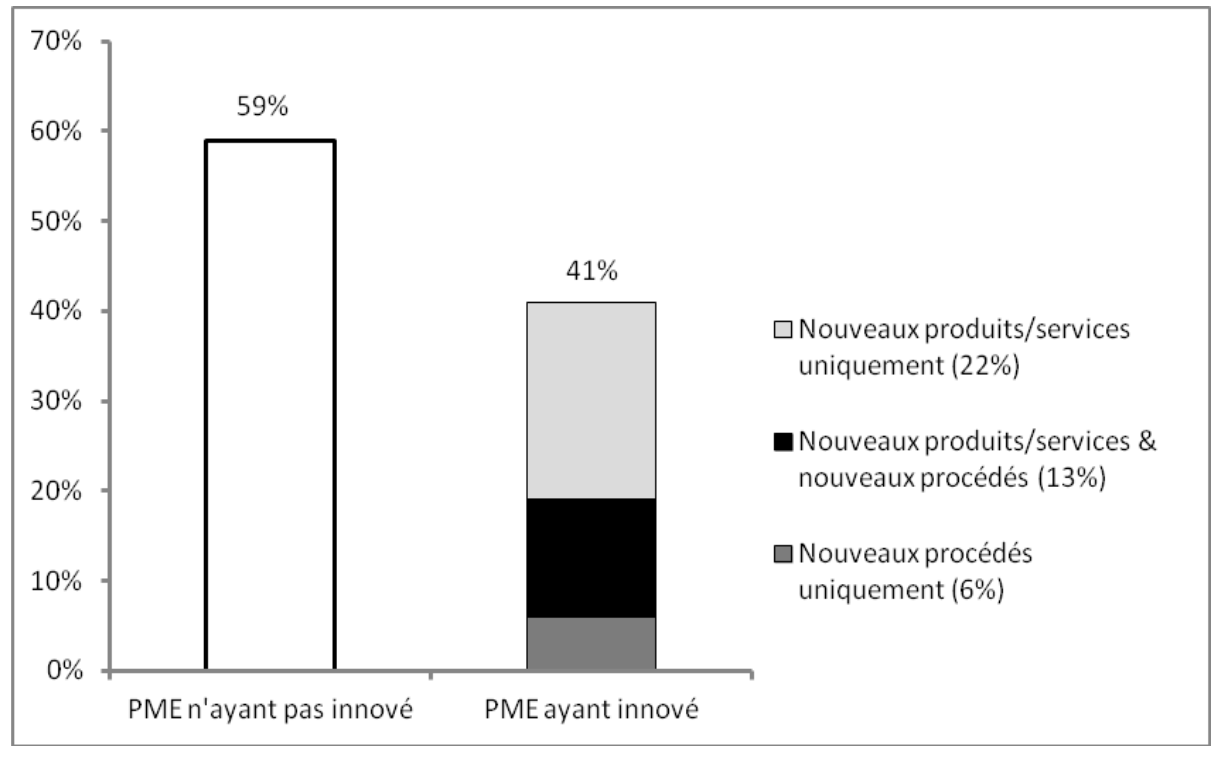

Figure 1 : Innovativité des PME de l'échantillon final (n=1086)

Parmi les entreprises innovantes, $27 \%$ ont accompagné leur innovation par un investissement dans un équipement TIC spécifique alors que $34 \%$ déclarent que l'innovation s'est accompagnée d'un usage plus intensif des TIC disponibles. Seules 79 PME innovantes (18\%) ont menés des efforts conjoints, en investissement et en usage des TIC. En définitive, c'est une majorité de PME innovantes (57\%) qui n'a pas accompagné ses innovations par les TIC (figure 2).

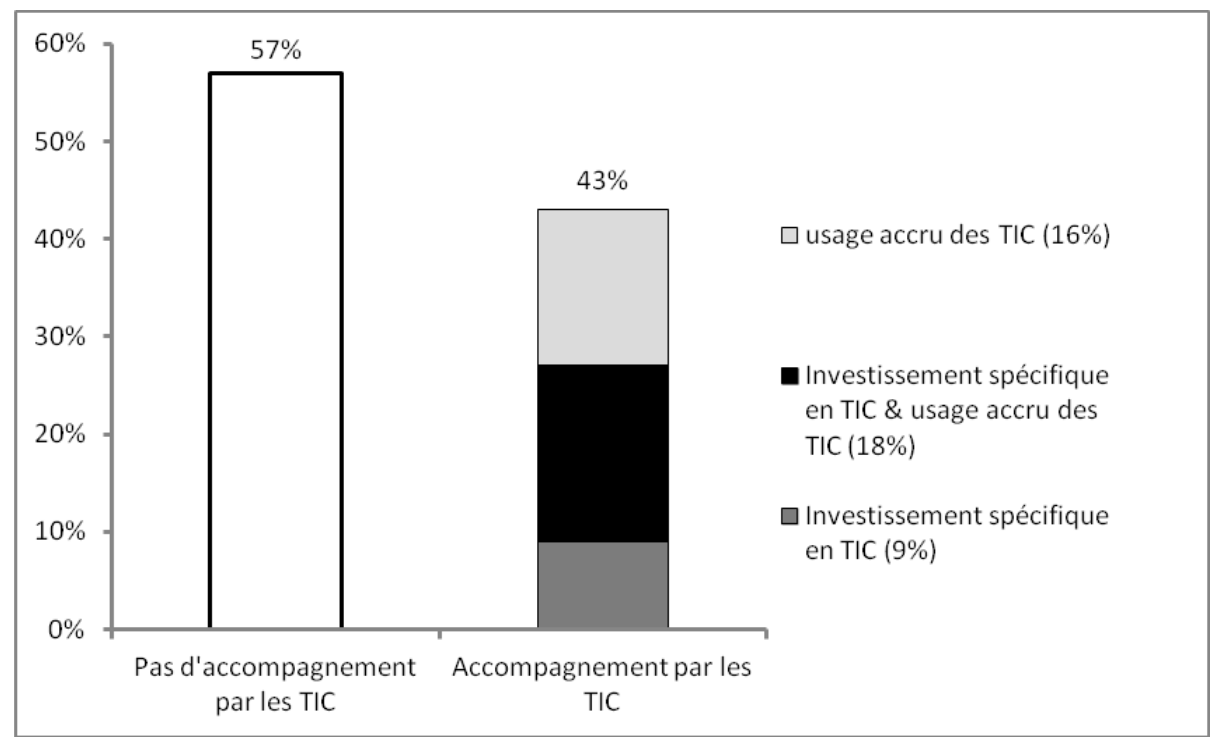

Figure 2 : Pratiques d'accompagnement par les TIC des innovations $(n=448)$ 


\section{III.1.2 La diversité des ressources TIC des PME}

Comme attendu, les éléments du questionnaire relatifs aux ressources TIC des PME montrent une diversité de situations, même si globalement le niveau général de ces ressources reste assez limité. Ainsi, les répondants déclarent majoritairement ne pas avoir de compétences informatiques internes $(62 \%)$ et seules $13 \%$ possèdent un service informatique constitué (figure 3).

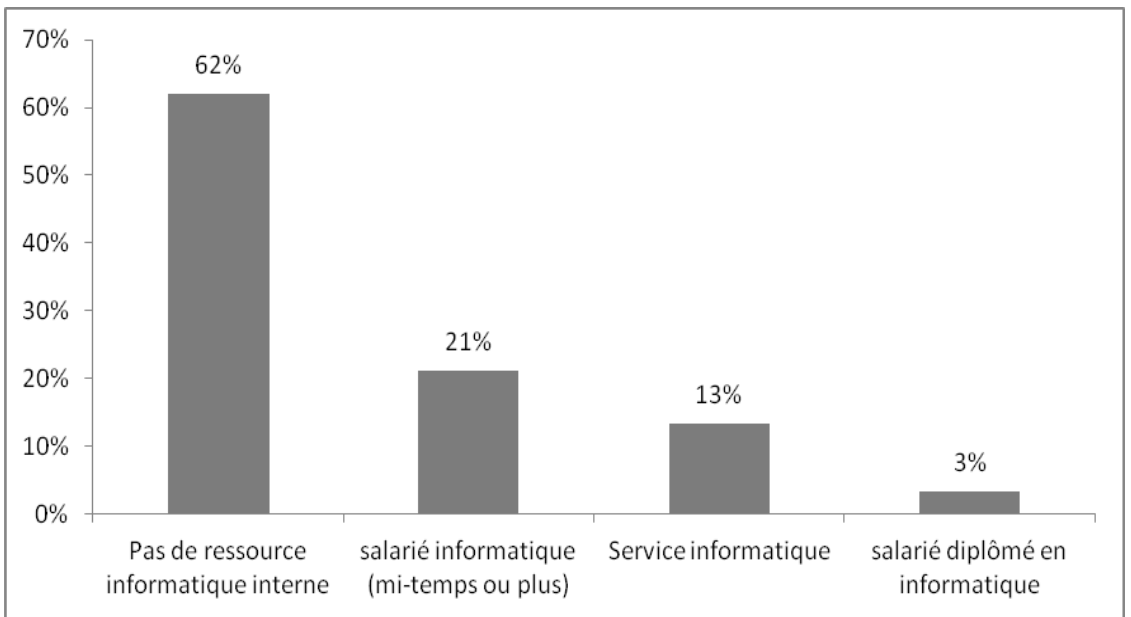

Figure 3 : Mesure des compétences informatiques internes aux PME (n=1086)

Concernant le niveau d'investissement en TIC perçu par les entreprises, la moitié (53\%) estime qu'elles investissent autant que la moyenne des firmes de leur secteur. 23\% des PME considèrent qu'elles investissent moins que la moyenne de leur secteur et $10 \%$ déclarent qu'elles investissent plus que la moyenne de leur secteur $(14 \%$ n'ont pas répondu à la question).

Malgré les ressources informatiques internes limitées, les PME ont fortement informatisé leurs services. Ainsi le taux moyen d'informatisation des fonctions approche $80 \%$ des services présents dans l'entreprise (moyenne à 0,78 et écart-type de 0,30 ).

Enfin, le taux d'adoption des applicatifs et des équipements montre une disparité de situations selon les outils considérés (figure 4). Un peu plus d'une PME sur deux utilise un logiciel métier spécifique ou dispose d'un site web informatif, alors que le taux d'adoption de la visioconférence ou des $\mathrm{ERP}^{2}$ reste marginal. Cette disparité d'équipement reflète des profils d'usage différenciés dans les petites entreprises, déjà mis en évidence dans des travaux antérieurs (Raymond et Bergeron, 2008).

\footnotetext{
${ }^{2}$ Un retraitement spécifique de la variable ERP a été mené afin de vérifier la présence effective du progiciel dans l'entreprise. Cette vérification est rendue nécessaire par le flou pouvant parfois entourer la notion d'ERP au sein des entreprises (Deltour et al., 2014). Sont retenues les PME déclarant avoir un ERP et ayant au moins deux fonctions utilisant un module ERP, dont la fonction comptable.
} 


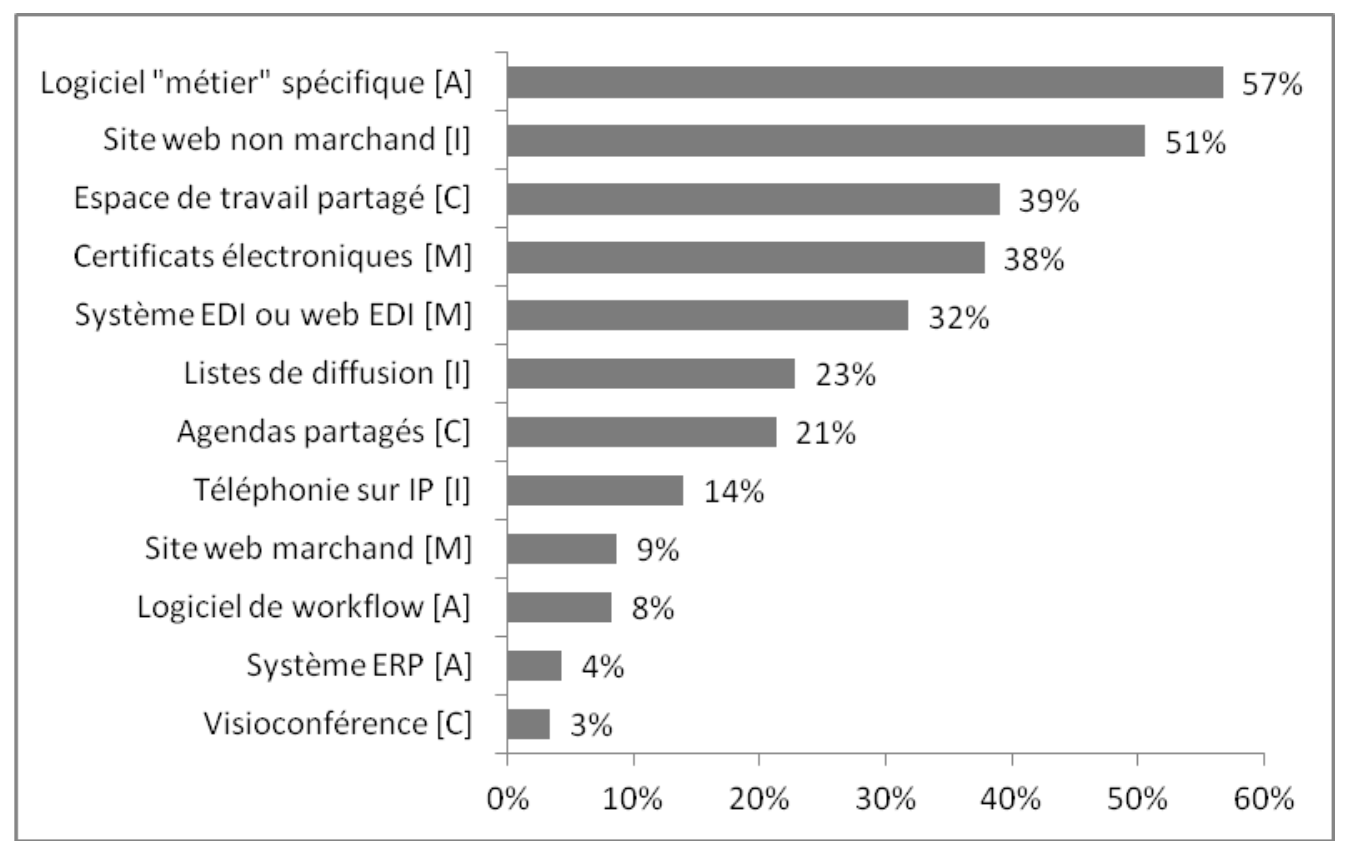

Figure 4 : Taux d'adoption ${ }^{3}$ des différents applicatifs/équipements dans les $\mathrm{PME}^{4}$

\section{III.1.3. La performance des PME}

Les données financières collectées en ligne indiquent que les entreprises de l'échantillon réalisent en moyenne $4442783 €$ de chiffre d'affaires, pour un taux de marge opérationnel de $3,4 \%$ pour l'année 2010. Le tableau 1 présente les résultats des entreprises (chiffre d'affaires et taux de marge opérationnel) dans trois sous-échantillons : celui des entreprises n'ayant pas innové, celui des entreprises ayant innové mais sans accompagner leur innovations par des TIC et enfin, le sous-échantillon des entreprises ayant innové et accompagné leurs innovations par des TIC.

\begin{tabular}{|l|l|l|l|}
\hline Echantillons & $\mathbf{N}$ & Chiffre d'Affaires moyen & $\begin{array}{l}\text { Taux de marge } \\
\text { d'exploitation moyen }\end{array}$ \\
\hline Echantillon total & 1086 & $4442783 €(\sigma=6148230)$ & $3,4 \%(\sigma=9,25)$ \\
\hline Entreprises non innovantes & 638 & $3906406 €(\sigma=5351277)$ & $3,65 \%(\sigma=9,17)$ \\
\hline $\begin{array}{l}\text { Entreprises ayant innové } \\
\text { sans accompagnement TIC }\end{array}$ & 256 & $4884403 €(\sigma=7044432)$ & $2,15 \%(\sigma=10,30)$ \\
\hline $\begin{array}{l}\text { Entreprises ayant innové } \\
\text { avec accompagnement TIC }\end{array}$ & 192 & $5523171 €(\sigma=7112144)$ & $4,33 \%(\sigma=7,82)$ \\
\hline
\end{tabular}

\section{Tableau 1 : Moyennes et écart-types des résultats financiers 2010 par sous-échantillon}

Ce tableau fait apparaître des écarts entre les trois sous-échantillons, la performance moyenne étant la plus importante au sein de l'échantillon des entreprises qui innovent et accompagnent leurs innovations par des TIC. Le chiffre d'affaires moyen est le plus faible pour les entreprises non innovantes, alors que le taux de marge moyen est plus faible pour les entreprises ayant innové sans accompagnement TIC que pour les non innovantes. Ces

\footnotetext{
${ }^{3}$ La base de calcul des taux d'adoption varie légèrement d'un applicatif/équipement à l'autre, du fait des non-réponses.

${ }^{4}$ Applicatifs/équipements orientés vers les quatre catégories : [M]archand, [I]nformation et communication, [C]ollaboration ou [A]utomatisation (cf. section 2.1.2).
} 
premiers résultats descriptifs ne permettent pour autant pas de conclure à un quelconque impact de la politique d'innovation des firmes et de leur accompagnement par les TIC sur leur performance.

\section{III.2. Analyse économétrique : les facteurs explicatifs de la performance}

Les données collectées nous permettent de tester l'effet sur les performances de l'accompagnement des innovations par les TIC. Conjointement, sont testés les effets sur la performance, d'une part de l'innovativité lorsque celle-ci n'est pas accompagnée par les TIC et d'autre part des ressources TIC présentes plus généralement dans l'entreprise. La mesure de ces effets est réalisée via la technique de la régression linéaire, en recourant à un modèle d'interaction (Wright, 1976 ; Braumoeller, 2004). Le principe consiste à intégrer dans la même régression, la variable d'interaction (Innovativité x Soutien TIC à l'innovation) et les variables simples (Brambor et al., 2005) : nous introduisons simultanément une variable simple indiquant si l'entreprise a ou non innové lors des deux dernières années, une variable d'interaction (prenant la valeur 1 lorsque l'entreprise a innové en accompagnant par des TIC et 0 sinon) ainsi que plusieurs variables mesurant les ressources TIC de l'entreprise. Enfin, le dynamisme du secteur et l'appartenance au secteur sont introduits dans la régression linéaire comme variables de contrôle.

Nous procédons à sept régressions successives incluant des mesures différentes des actifs TIC, permettant un affinement de la manière dont sont appréhendées ces actifs : perception du niveau d'investissement général en TIC (modèle 1), taux d'informatisation des fonctions (modèle 2), niveau d'adoption des applicatifs (modèle 3 ) et finalement niveau d'adoption de différentes catégories d'applicatifs (modèles 4 à 7).

Le tableau 2 présente les coefficients des différentes variables (hors variables de contrôle), ainsi que le degré de significativité des variables (une étoile pour $10 \%$, deux pour $5 \%$ et trois pour $1 \%$ ). Pour les variables multinomiales, la modalité de référence est notée «Réf». 


\begin{tabular}{|c|c|c|c|c|c|c|c|c|}
\hline \multicolumn{2}{|c|}{ Variables explicatives } & Modèle 1 & Modèle 2 & Modèle 3 & Modèle 4 & Modèle 5 & Modèle 6 & Modèle 7 \\
\hline \multicolumn{2}{|c|}{ Innovativité } & $-1.56 * *$ & $-1,55 * *$ & $-1,43 * *$ & $-1,51 * *$ & $-1,50 * *$ & $-1,50 * *$ & $-1,56 * *$ \\
\hline \multicolumn{2}{|c|}{$\begin{array}{l}\text { Innovativité } \\
\text { x Soutien TIC à l'innovation }\end{array}$} & $2.13 * *$ & $1,86 * *$ & $2,31 * * *$ & $2,07 * *$ & $2,24 * * *$ & $2,32 * * *$ & $2,08 * *$ \\
\hline \multirow{5}{*}{ 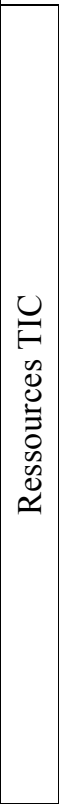 } & $\begin{array}{r}\text { Compétences informatiques } \\
\text { Service constitué } \\
\text { Salarié dédié } \\
\text { Salarié diplômé non dédié } \\
\text { Aucune de ces compétences } \\
\text { internes }\end{array}$ & $\begin{array}{r}-0,15 \\
-0,37 \\
2,34 \\
\text { Réf. }\end{array}$ & $\begin{array}{r}-0,04 \\
-0,30 \\
2,02 \\
\text { Réf. }\end{array}$ & $\begin{array}{r}0,32 \\
-0,22 \\
2,38 \\
\text { Réf. }\end{array}$ & $\begin{array}{r}-0,01 \\
-0,30 \\
2,26 \\
\text { Réf. }\end{array}$ & $\begin{array}{r}0,14 \\
-0,26 \\
2,39 \\
\text { Réf. }\end{array}$ & $\begin{array}{r}0,18 \\
-0,32 \\
2,35 \\
\text { Réf. }\end{array}$ & $\begin{array}{r}0,09 \\
-0,29 \\
2,26 \\
\text { Réf. }\end{array}$ \\
\hline & $\begin{array}{l}\text { Investissement en TIC } \\
\text { Supérieur à la moyenne } \\
\text { Dans la moyenne } \\
\text { Inférieur à la moyenne } \\
\end{array}$ & $\begin{array}{c}-1,66 * \\
\text { Réf } \\
-1,02 \\
\end{array}$ & & & & & & \\
\hline & Taux d'informatisation & & $-1,96 * *$ & & & & & \\
\hline & Adoption des applicatifs & & & $-0,35 * * *$ & & & & \\
\hline & $\begin{array}{l}\text { Adoption des applicatifs : } \\
\text { - Marchands } \\
\text { - d'Information } \\
\text { - de Collaboration } \\
\text { - d'Automatisation } \\
\end{array}$ & & & & $-0,61 *$ & $-0.70 * *$ & $-0,82 * *$ & $-0,59$ \\
\hline \multicolumn{2}{|l|}{$\mathrm{R}^{2}$} & 0,058 & 0,059 & 0,059 & 0,06 & 0.057 & 0,058 & 0,055 \\
\hline \multicolumn{2}{|c|}{ Observations utiles } & 1086 & 1040 & 1086 & 1086 & 1086 & 1086 & 1086 \\
\hline
\end{tabular}

Tableau 2 : Facteurs explicatifs de la performance des PME (taux de marge d'exploitation en 2010)

Les résultats des modèles économétriques du tableau 2 indiquent que le coefficient de la variable d'interaction est positif et significatif, ce qui nous conduit à valider l'hypothèse selon laquelle l'accompagnement de l'innovation par les TIC contribue à la performance des PME : les entreprises innovatrices qui accompagnent leur innovation par les TIC ont des performances plus importantes que les innovatrices qui ne mobilisent pas les TIC. Globalement, les firmes qui innovent et accompagnent leur innovation par les TIC ont des performances plus importantes que celles qui n'innovent pas, même si cette différence est faible : elle est en effet donnée par la somme des coefficients de la variable simple et de la variable d'interaction (de l'ordre de 0.6 à 0.88 selon les modèles) (Brambor et al., 2005). En revanche, le coefficient de la variable simple - significatif et négatif - nous indique que l'innovativité non accompagnée par les TIC a un effet négatif sur la performance des firmes. Ce résultat n'est pas intuitif au regard de la littérature existante. Il est intéressant de noter que ces résultats concernant l'innovativité et son accompagnement par les TIC sont stables dans les sept modèles testés.

L'effet des ressources TIC non directement affectées à l'innovation est également inattendu : si la présence de compétences internes en informatique n'affecte pas la performance, les coefficients des autres variables mesurant les actifs TIC de manière globale traduisent un effet négatif de l'informatisation de l'entreprise sur sa performance. Les effets sont plus complexes lorsqu'on distingue les actifs TIC en fonctions des objectifs auxquels ils répondent dans l'entreprise, mais l'effet sur la performance n'est jamais positif.

Les coefficients des variables de contrôle (non présentés dans le tableau) traduisent un effet environnement et un effet sectoriel : les PME dont le marché principal est en croissance ont une meilleure performance que celles caractérisées par un marché principal stable; la 
décroissance du marché principal ne semble en revanche pas significativement affecter le taux de marge des PME. De façon complémentaire, les modèles font apparaître des effets sectoriels, certains secteurs d'activité étant caractérisés par des taux de marge inférieurs ou supérieurs au secteur de référence.

Enfin, si le coefficient de détermination des modèles $\left(\mathrm{R}^{2}\right)$ est assez faible, cela peut s'expliquer par le choix d'une mesure objective de la performance comme variable dépendante. La valeur obtenue est comparable à celle de travaux ayant adopté une approche similaire (Aral et Weill, 2007 ; Huang et Liu, 2005).

\section{III.3. Discussion}

Les résultats présentés ci-dessus ne confirment que partiellement les résultats admis dans la littérature. Ils apportent donc des conclusions inattendues sur la relation entre innovativité, TIC et performance, qu'il est nécessaire de discuter.

\section{III.3.1. L'innovativité soutenue par un accompagnement numérique ciblé est source de performance}

Nos investigations valident l'hypothèse d'un effet combiné de l'innovativité et des TIC renforçant la performance, élargissant aux PME des conclusions déjà mises en évidence sur des grandes entreprises (Huang et Liu, 2005). Notre investigation confirme et élargit donc les résultats des travaux portant sur l'utilisation de certaines technologies spécifiques comme soutien aux activités d'innovation (Pavlou et El Sawy, 2006 ; Banker et al., 2006 ; Tambe et al., 2012). Sans restreindre notre étude à des technologies déterminées ou des types d'innovations spécifiés, nous mettons en évidence un effet positif de l'innovativité des PME sur leur performance, dès lors qu'elles mobilisent des TIC pour accompagner leurs innovations, que ce soit en produits ou en procédés. On peut alors parler d'effet de synergie ciblé entre TIC et innovativité.

De plus, nous montrons que l'effet positif de l'innovation est conditionné par un accompagnement de ces innovations suite à des investissements spécifiques dans les TIC. Ce résultat va dans le sens des travaux de Dibrell et al. (2008) qui montrent que l'impact positif de l'innovation passe nécessairement par des investissements dans les TIC. Mais il est aussi intéressant de noter que l'accompagnement des innovations par des TIC ne s'entend pas uniquement en termes d'investissement : un usage plus développé des technologies présentes dans l'entreprise est considéré selon notre approche comme un accompagnement et contribuera positivement à la performance. Cette perspective élargie des TIC s'appuie sur l'idée de divergences d'appropriation et d'usage des TIC d'une entreprise à l'autre, notamment dans les PME (Saga et Zmud, 1996).

\section{III.3.2. L'engagement dans l'innovation ou les TIC minorent les performances des PME}

Un résultat plus inattendu est celui des effets de l'innovativité et des ressources TIC, considérées isolément. Nous montrons que les entreprises qui déclarent avoir innové dans les deux dernières années mais ne pas avoir mobilisé les TIC pour accompagner ces innovations ont une moins bonne performance que les entreprises n'ayant pas innové. De même les ressources TIC, si elles ne sont pas mobilisées spécifiquement pour accompagner une stratégie d'innovation ont un effet nul ou négatif sur la performance des PME. L'alignement de la politique de l'entreprise en termes de TIC à sa stratégie d'innovation semble donc 
nécessaire pour optimiser les investissements réalisés ou les actifs mobilisés dans ces deux sphères.

Certaines recherches antérieures ont montré que le lien entre innovativité et performance n'est pas systématiquement positif. En particulier, dès lors que les recherches intègrent une possible interaction entre TIC et innovativité, l'effet direct de cette dernière sur la performance devient plus complexe (Dibrell et al., 2008), voire non linéaire (Huang et Liu, 2005). Pour expliquer ce phénomène, Huang et Liu s'appuient sur l'idée de rendements décroissants de la R\&D. Nos résultats sont plus tranchés : une innovation non accompagnée par les TIC minore la performance. Une piste d'explication de ce résultat porte sur les difficultés en termes de retour sur investissement pour les activités d'innovation dans les PME: la mise en œuvre d'innovations a un coût significatif pour l'organisation, mais les bénéfices retirés restent incertains au niveau commercial ou organisationnel. Ainsi, comme le rappelle Subramanian (1996), les entreprises classées dans la catégorie des «prospecteurs » (selon la typologie de Miles et Snow) sont de manière générale les plus innovantes, mais ne sont pas nécessairement les plus performantes d'un point de vue financier. Le retour sur investissement de l'innovation sera donc d'autant plus difficile que les outils TIC disponibles dans l'entreprise ne sont pas mobilisés pour accompagner ces innovations ou que les éventuels investissements technologiques réalisés ne viennent pas en support de ces innovations.

Le lien entre ressources TIC et performance est lui aussi questionné. Nos travaux montrent que seules les TIC reliées aux innovations contribuent positivement à la performance. La présence de compétences en informatique au sein de l'entreprise n'a en effet pas d'impact direct sur la performance, alors que les différentes variables mesurant les ressources matérielles en TIC ont de manière globale un effet négatif sur cette même performance, et ce quelle que soit la mesure utilisée. En effet, que les actifs TIC soient mesurés par l'investissement global perçu de l'entreprise, par le taux d'informatisation des fonctions ou par la somme des applicatifs utilisés, l'effet sur la performance reste négatif à des degrés de significativité plus ou moins importants. Ces résultats alimentent l'idée que les TIC ne sont plus des sources d'avantages concurrentiels (Carr, 2003).

La distinction des actifs TIC par catégories, en fonction de leurs finalités, aboutit à une analyse assez faiblement différenciée : sur les quatre catégories d'applicatifs considérées, trois ont un effet négatif sur la performance (applicatifs d'information, applicatifs de collaboration et applicatifs marchands) alors que la quatrième catégorie regroupant les applicatifs d'automatisation (logiciels métier, logiciels de workflow et ERP) n'a pas d'effet significatif. Cette dernière catégorie se distingue des autres du fait que les applicatifs considérés sont directement centrés sur les gains d'efficience et la réduction des coûts dans l'activité productive de entreprise (Deltour et al., 2014); le surcoût engendré par l'adoption de ces applicatifs semble donc compensé par le gain en efficacité, ce qui ne serait pas le cas pour les autres catégories d'applicatifs. Le fait d'avoir réalisé notre investigation auprès de PME peut participer à expliquer ces résultats : de même que les stratégies d'innovation sont coûteuses pour les PME, le coût de l'informatisation est perçu comme élevé par ces entreprises (Monnoyer et Boutary, 2008). De plus, le gain stratégique et financier des TIC n'est pas évident pour des petites structures dont le numérique n'est pas le cœur d'activité, d'autant que ces technologies ont tendance à devenir rapidement obsolètes et qu'elles permettent de moins en moins facilement de se distinguer des autres firmes (Chae et al., 2014). Seuls des investissements, matériels ou humains, dans les TIC visant à accompagner une initiative stratégique de la firme (réorganisation, innovation, etc.) auront un effet positif sur sa performance. Les coûts supportés par des petites structures dans le cadre de leurs activités d'innovation ou de leur politique en termes de TIC apparaissent comme élevés au regard de leur taille. Donc, si ces dépenses ne sont pas réalisées pour répondre à une ambition 
organisationnelle spécifique, elles risquent d'induire des coûts qui ne seront pas compensés par des retombées positives suffisantes. D'une manière générale, nos résultats font donc écho aux travaux sur la valeur des technologies de l'information (Melville et al., 2004) et ses limites (Carr, 2003) en accentuant certaines conclusions : la valeur des TIC a été montrée comme d'autant plus forte qu'elle est combinée à des capacités organisationnelles (Liang et al. 2010) ; notre investigation montre que la combinaison à une capacité organisationnelle comme la capacité d'innovation est une condition même de création de valeur par les technologies de l'information en PME.

Nos résultats ont des implications pour tous les acteurs concernés par les activités touchant au numérique, en particulier à l'intérieur de l'organisation (dirigeants, responsables informatiques, etc.) : le choix de s'équiper ou de renforcer l'usage des TIC dans la PME n'a de sens que lorsqu'il est fait en cohérence avec sa politique générale, notamment sa politique d'innovation de l'entreprise. Cette implication n'a rien d'évident dans un contexte de faible acculturation numérique de nombreux acteurs en PME. La faiblesse constatée des compétences informatiques internes incite d'ailleurs à ce que d'autres acteurs hors de l'organisation tiennent compte aussi de ces enseignements. Il s'agit notamment des acteurs publics (par leurs politiques de soutien à l'innovation et de soutien à l'équipement en TIC), ainsi que les consultants (par leur activité de conseil) : pouvoirs publics et sociétés de conseil ont tout intérêt à sensibiliser les PME au fait que toute activité innovante (nouveaux produits, nouveaux procédés) doit s'accompagner d'un volet numérique, afin que l'innovation engagée puisse porter ses fruits.

\section{III.3.3. Enseignements méthodologiques}

Les résultats obtenus dans notre recherche sont à mettre en relation avec les choix méthodologiques retenus. Ces choix diffèrent pour partie des options prises dans les travaux antérieurs. Nous réaffirmons ici les choix effectués et leurs apports pour l'analyse du lien des TIC et de l'innovativité à la performance.

Tout d'abord nous mobilisons une mesure large de l'innovativité (Subramanian, 1996 ; Wang et Ahmed, 2004), qui peut inclure des stratégies visant dans certaines entreprises à rattraper un éventuel retard, à se remettre à niveau par rapport à leur concurrentes. Cela constitue une différence fondamentale avec les travaux qui mesurent l'innovativité par le niveau de R\&D ou de dépôt de brevets par l'entreprise (Kleis et al., 2012).

De plus, la mesure de l'accompagnement par les TIC retenue dans notre recherche diffère de l'approche généralement retenue : une synergie supposée d'investissements simultanés en R\&D et en TIC (Huang et Liu, 2005 ; Kmieciack et al., 2012 ; Raymond et al., 2013). En effet, rien ne dit que dans la pratique ces investissements sont reliés, c'est-à-dire qu'il y ait croisement effectif de ces deux efforts. Dans notre cas, les TIC dédiées à l'innovation traduisent des investissements technologiques ou des usages induits spécifiquement par l'innovation, ce qui constitue un apport au regard de la littérature existante.

Par ailleurs, la mesure des TIC est parfois faite via un montant financier (Huang et Liu, 2005 ; Dibrell et al., 2008), alors qu'un usage plus développé des technologies existantes constitue aussi un accompagnement par les TIC. L'innovativité non accompagnée par les TIC est donc plus restrictive dans notre étude que dans les travaux existants, ce qui peut expliquer l'existence des effets directs négatifs. L'un des résultats de notre article est donc que, si l'accompagnement des innovations par les TIC est nécessaire pour améliorer la performance des entreprises, cet accompagnement n'a pas nécessairement de contrepartie financière. 
Concernant les ressources TIC, notre méthode s'écarte de l'estimation financière d'un budget informatique global. En suivant l'approche de Melville et al. (2004) ou Aral et Weill (2007), nous faisons le choix de considérer, d'une part, les ressources humaines en informatique et, d'autre part, les ressources matérielles en TIC (mesurées de trois manières différentes). Nous faisons apparaître un effet différencié, la présence de compétences informatiques internes à l'entreprise n'ayant pas d'effet sur la performance, alors que le niveau des actifs matériels, quelle que soit la mesure utilisée, a lui un effet négatif.

Enfin, il est intéressant de rappeler que nous nous sommes appuyés sur des mesures objectives de la performance, pratique méthodologique moins usuelle car souvent plus difficile à mettre en œuvre (Tallon et Kraemer, 2007 ; Rougès et al., 2010). Retenant une double mesure de la performance, Kmieciak et al. (2012) concluent que la corrélation entre l'innovativité et la performance est supérieure dans le cas de mesures subjectives, suggérant que la subjectivité amplifie la perception des liens analysés.

\section{Conclusion}

L'innovativité, en tant que capacité à s'engager dans l'innovation, constitue un trait essentiel des organisations, pouvant toucher aussi bien les petites que les grandes entreprises. Parallèlement, la montée en puissance des technologies de l'information concerne l'ensemble des entreprises quelle que soit leur taille. Le rôle de ces technologies est multiple au sein des organisations, ce qui induit des appropriations et des usages disparates, notamment dans les petites structures (Saga et Zmud, 1996 ; Raymond et Bergeron, 2008 ; Monnoyer et Boutary, 2008). Dans cette recherche, nous nous sommes interrogés sur les effets de l'accompagnement numérique de l'innovation. Les résultats, issus d'une analyse sur 1086 PME, sont riches en enseignements pour les responsables de petites entreprises. En effet, notre étude montre la nécessité pour ces entreprises d'accompagner leurs stratégies d'innovation (en produits ou en procédés) par des investissements dans les TIC ou par un renforcement de l'utilisation des technologies déjà présentes. A l'inverse, sans accompagnement numérique, ces entreprises s'exposent à ce que leur politique d'innovation se solde par une contraction de leurs performances financières. Parallèlement, la présence de compétences en informatique au sein de l'entreprise (par exemple disposer d'un service informatique ou un salarié informaticien) apparait comme neutre vis-à-vis du niveau de performance global de l'entreprise, alors qu'une politique d'équipement intensif en actifs TIC (fort investissement général en TIC, taux élevé d'informatisation des fonctions ou bien déploiement de nombreux applicatifs) a un effet négatif sur la performance.

Si l'effet positif induit par la combinaison des stratégies d'innovation et d'utilisation des TIC sur la performance des firmes a déjà été démontré dans la littérature (Huang et Liu, 2005, Dibrell et al., 2012), la mise en évidence d'un effet négatif des stratégies d'innovativité et des ressources matérielles en TIC des PME sur leurs performances constitue un résultat novateur. L'ensemble de nos résultats est soutenu par des choix méthodologiques originaux, mais appropriés au sujet étudié : l'innovativité est considérée au sens large ; l'accompagnement par les TIC est mesuré de façon directe, passant à la fois par des investissements spécifiques ou par un usage plus développé des TIC. Ce dernier point constitue une différence fondamentale avec les travaux existants, dans lesquels l'accompagnement est le plus souvent mesuré par des investissements concomitants en R\&D et dans les TIC.

L'intérêt des résultats actuels laisse cependant certaines questions en suspens. La distinction entre les retombées à attendre des innovations de produits et de procédés (Forsman, 2011) est une piste qui reste à explorer : les innovations de produits sont souvent plus risquées et 
correspondent à un changement fort sur le marché voire à une création de marché, alors que les innovations de procédé visent à tirer bénéfice d'une amélioration dans la fabrication du bien ou du service avec, la plupart du temps, un moindre engagement financier. Une seconde piste porte sur la distinction entre les deux formes d'accompagnement des innovations par les TIC. En effet, le recours à des investissements spécifiques et l'utilisation de ressources existantes pourraient avoir des effets différenciés sur la performance. Ces différents éléments constituent alors autant d'axes pour prolonger la recherche actuelle afin de mieux comprendre et mesurer la manière dont les TIC et l'innovation se conjuguent et peuvent bénéficier aux PME.

\section{Remerciements}

Nous remercions le Groupement d'Intérêt Scientifique (GIS) M@rsouin (http://www.marsouin.org/) ainsi que la Chambre de Commerce et d'Industrie de la Région Bretagne pour l'enquête menée dans le cadre de l'observatoire OPSIS (Observation et Prospective sur la Société de l'Information et ses Services) dédié aux usages du numérique en Bretagne.

\section{Références}

Aral, S. \& Weill P. (2007), "IT Assets, Organizational Capabilities, and Firm Performance: How Resource Allocations and Organizational Differences Explain Performance Variation, Organization Science, Vol. 18, n5, p. 763-790.

Banker, R.D., Bardhan, I. \& Asdemir, O. (2006), "Understanding the Impact of Collaboration Software on Product Design and Development", Information Systems Research, Vol. 17, n4, p. 352-373.

Besson, P. \& Rowe, F. (2011), "Perspectives sur le phénomène de la transformation organisationnelle », Systèmes d'Information et Management, Vol. 16, n¹, p. 3-34.

Bharadwaj, A. (2000), "A Resource-based Perspective on the Information Technology Capability and Firm Performance: An Empirical Investigation." MIS Quarterly, Vol. 24, n 1 , p. 169-196.

Brambor, T., Clark W. \& Golder M. (2005), "Understanding Interaction Models: Improving Empirical Analyses", Political Analysis, Vol. 13, p. 1-20.

Braumoeller, B. (2004), "Hypothesis Testing and Multiplicative Interaction Terms", International Organization, Vol. 58, n ${ }^{\circ}$, p. 807-820.

Cardona, M., Kretschmer, T. \& Strobel T. (2013), "ICT and Productivity: Conclusions from the Empirical Literature”, Information Economics and Policy, Vol. 25, n³, p. 109-125.

Carr, N. (2003), “IT doesn’t matter”, Harvard Business Review. Vol. 81, n5, p. 41-49.

Chae, H.C., Koh, C. \& Pributok, V. (2014), "Information Technology Capability and Firm Performance: Contradictory Findings and their Possible Causes", MIS Quarterly, Vol. 38, $\mathrm{n}^{\circ} 1$, p. 305-326.

Cigref (2013), «La contribution de l'IT à l'innovation - les facteurs constitutifs des démarches d'innovation », Etude, 27 pages. 
Damanpour, F. (1991), "Organizational Innovation: A Meta-analysis of Effects of Determinants and Moderators", The Academy of Management Journal, Vol. 34, n³, p. 555590.

Das, S. \& Joshi M. (2012), "Process Innovativeness and Firm Performance in Technology Service Firms: The Effect of External and Internal Contingencies", IEEE Transactions on Engineering Management, Vol. 59, n³, p. 401-414.

Deltour, F., Farajallah, M. \& Lethiais, V. (2014) « L'équipement des PME en systèmes ERP : une adoption guidée par les priorités stratégiques? », Management International, Vol. 18, $\mathrm{n}^{\circ} 2$, p. $155-168$.

Dibrell, C., Davis, P. \& Craig, J. (2008), "Fueling Innovation through Information Technology in SMEs", Journal of Small Business Management, Vol. 46, n², p. 203-218.

Foray, D. \& Mairesse, J. (eds) (1999), Innovations et performances - approches pluridisciplinaires, Editions de l'EHESS, 470 pages.

Forsman, H. (2011), "Innovation Capacity and Innovation Development in Small Enterprises - A Comparison between the Manufacturing and Service Sectors", Research Policy, Vol. 40, n5, p. 739-750.

Hadjimanolis, A. (2000), "A Resource-based View of Innovativeness in Small Firms", Technology Analysis \& Strategic Management, Vol. 12, n², p. 263-281.

Hausman, A. (2005), "Innovativeness among Small Businesses: Theory and Propositions for Future Research", Industrial Marketing Management, Vol. 34, n8, p. 773-782.

Huang, C.J. \& Liu, C.J. (2005), "Exploration for the Relationship between Innovation, IT and Performance” Journal of Intellectual Capital, Vol. 6, n² 2, p. 237-252.

Hult, G., Hurley, R. \& Knight, G. (2004), "Innovativeness: Its Antecedents and Impact on Business Performance”, Industrial Marketing Management, Vol. 33, n5, p. 429-438.

Jansen, J., Van Den Bosch, F. \& Volberda, H. (2006), “Exploratory Innovation, Exploitative Innovation, and Performance: Effects of Organizational Antecedents and Environmental Moderators", Management Science, Vol. 52, n¹1, p. 1661-1674.

Kafouros, M. \& Wang, C. (2008), "The Time Lag between R\&D Investments and their Effects on Performance", Industry and Innovation, Vol. 15, n³, p. 233-151.

Kafouros, M. (2005), "R\&D and Productivity Growth: Evidence from the UK", Economics of Innovation and New Technology, Vol. 14, nº, p. 479-497.

Kleis, L., Chwelos, P., Ramirez, R. \& Cockburn, I. (2012), "Information Technology and Intangible Output: The Impact of IT Investment on Innovation Productivity", Information Systems Research, Vol. 23, n¹, p. $42-59$.

Kmieciak, R., Michna, A. \& Meczynska, A., (2012), "Innovativeness, Empowerment and IT Capability: Evidence from SMEs”, Industrial Management \& Data Systems, Vol. 112, n5, p. 707-728.

Kohli, R. \& Devaraj, S., (2003), "Measuring Information Technology Payoff: A MetaAnalysis of Structural Variables in Firm-Level Empirical Research", Information Systems Research, Vol. 14, n², p. 127-145.

Kuusisto, A. \& Riepula, M. (2011), "Customer Interaction in Service Innovation: Seldom Intensive but often Decisive. Case Studies in three Business Service Sectors", International Journal of Technology Management, Vol. 55, $\mathrm{n}^{\circ} 1 / 2$, p. 171-186. 
Liang, T.P., You, J.J. \& Liu, C.C. (2010), "A Resource-based Perspective on Information Technology and Firm Performance: a Meta Analysis", Industrial Management \& Data Systems, Vol. 110, nº 8 , p. 1138-1158.

Loilier, T. \& Tellier, A. (2013), Gestion de l'innovation, $2^{\text {ème }}$ édition, éditions EMS, 527 pages.

Marchesnay, M. (1991), «La PME : une gestion spécifique », Économie Rurale, n 206, p. 11-17.

Marsouin (2009), "Pénétration, diffusion et usages des TIC dans les PME », Document en ligne disponible à l'adresse : http://www.marsouin.org/spip.php?article292, 15 pages.

Melville, N., Kraemer, K. \& Gurbaxani, V., (2004), "Review - Information Technology and Organizational Performance: an Integrative Model of IT Business Value", MIS Quarterly, Vol. 28, n², p. 283-322.

Menguc, B. \& Auh, S. (2006), "Creating a Firm-level Dynamic Capability through Capitalizing on Market Orientation and Innovativeness", Journal of the Academy of Marketing Science, Vol. 34, n 1 , p. 63-73.

Merminod, V., Mothe, C. \& Rowe, F. (2009), «Effets de Product Lifecycle Management sur la fiabilité et la productivité : une comparaison entre deux contextes de développement produit»,M@n@gement, Vol.12,n4,p.294-331.

Monnoyer, M.C. \& Boutary, M. (2008), «Constats et hypothèses pour mieux comprendre l'appropriation des TIC en PME », Analyser les usages des systèmes d'information et des TIC, Quelles démarches, quelles méthodes? (M. Benedetto-Meyer et R. Chevallet, eds), éditions ANACT, p. 98-125.

Oseo (2011), «La défaillance des entreprises - Étude sur données françaises entre 2000 et $2010 »$, Regards sur les PME, n²1, éditions La Documentation Française.

Pavlou, P. \& El Sawy, O. (2006), "From IT Leveraging Competence to Competitive Advantage in Turbulent Environments: the Case of New Product Development", Information Systems Research, Vol. 17, n³, p. 198-227.

Poulin, D. \& Tran, S. (eds.), (2010), Information and Communication Technology and Small and Medium Sized Enterprises: From Theory to Practice, Cambridge Scholars publishing, 192 pages.

Premkumar, G. (2003), "A Meta-analysis of Research on Information Technology Implementation in Small Business", Journal of Organizational Computing and Electronic Commerce, Vol. 13, n², p. 91-121.

Raymond, L. \& Bergeron, F. (2008), "Enabling the Business Strategy of SMEs through ebusiness Capabilities: A Strategic Alignment Perspective", Industrial Management \& Data Systems, Vol. 108, n 5, p. 577-595.

Raymond, L., Bergeron, F. \& Croteau, A.M. (2013), "Innovation Capability and Performance of Manufacturing SMEs: The Paradoxical Effect of IT Integration", Journal of Organizational Computing and Electronic Commerce, Vol. 23, n³, p. 249-272.

Rougès, J.F., Poulin, D., D’Amours, S. \& Montreuil, B. (2010), "Relationship between SME Performance and Information and Communication Technology", in Information and Communication Technology and Small and Medium Sized Enterprises: From Theory to Practice (Poulin D. and Tran S. eds.), Cambridge Scholars publishing, p. 25-47. 
Saga, V. \& Zmud, R. (1996), «Introduction de logiciels de gestion dans des petites entreprises liées à une profession libérale », Systèmes d'Information et Management, Vol. 1, $\mathrm{n}^{\circ} 1$, p. 51-73.

Subramanian, A. (1996), "Innovativeness: Redefining the Concept", Journal of Engineering \& Technology Management, Vol. 13, $\mathrm{n}^{\circ}$ 3/4, p. 223-243.

Tallon, P. \& Kraemer, K. (2007), "Fact or Fiction? A Sensemaking Perspective on the Reality behind Executives Perceptions of IT Business Value", Journal of Management Information Systems, Vol. 24, n 1, p. 13-54.

Tambe, P., Hitt, L. \& Brynjolfsson, E. (2012), "The Extroverted Firm: How External Information Practices Affect Innovation and Productivity", Management Science, Vol. 58, n5, p. 843-859.

Wang, C.L. \& Ahmed, P.K. (2004), "The Development and Validation of the Organisational Innovativeness Construct using Confirmatory Factor Analysis", European Journal of Innovation Management, Vol. 7, n 4, p. 303-313.

Wright, G. (1976), "Linear Model for Evaluating Conditional Relationships", American Journal of Political Science, vol. 20, n², p. 349-373.

\section{Annexe : extraits du questionnaire}

Les modalités de réponses aux questions sont indiquées entre crochets. Les répondants disposaient en fin de questionnaire d'un glossaire définissant certains termes et acronymes pouvant poser des difficultés de compréhension.

\section{Innovativité}

Dans les 24 derniers mois, votre entreprise... s'est positionnée sur de nouveaux produits/services [oui/non] / a introduit de nouveaux procédés [oui/non]

\section{Soutien TIC à l'innovation}

S'il y a eu innovations (produit ou procédé), cela s'est traduit par... un investissement dans un équipement TIC spécifique [oui/non] / un usage plus développé des TIC [oui/non]

\section{Compétences informatiques internes}

Parmi ces situations, lesquelles s'appliquent à votre entreprise [oui/non pour chaque item] : Il y a un service informatique constitué / Il y a au moins un salarié à mi-temps ou plus dédié à l'informatique / Il y a au moins un salarié diplômé du supérieur en informatique dans l'entreprise

\section{Investissement agrégé en TIC}

Diriez-vous que l'investissement de votre entreprise dans les TIC (informatique, internet, outils collaboratifs, etc.) est [réponse au choix] : supérieur à la moyenne de votre secteur / dans la moyenne de votre secteur / inférieur à la moyenne de votre secteur.

\section{Informatisation des fonctions}

Comment votre entreprise assure-t-elle la gestion de ces différentes fonctions ? [tableau croisé]

(Horizontalement) Liste de fonctions: comptabilité ou finance, gestion des ventes, prospection et actions commerciales, gestion des achats, gestion des stocks, planification et 
gestion de production, logistique/distribution, maintenance, contrôle de gestion, qualité, ressources humaines, décisionnel (pilotage, business intelligence).

(Verticalement) Si fonction existant en interne : non informatisée / avec des logiciels du marché (progiciels) / avec un module d'un ERP/PGI / avec une solution maison (développée en interne). Sinon : fonction externalisée / fonction non existante / ne sais pas.

\section{Adoption des applicatifs}

Dans votre entreprise, utilise-t-on [oui/non pour chaque item]: un système informatisé d'échange de données (EDI ou Web EDI) / des listes de diffusion internes / des agendas partagés / des espaces de travail partagés (logiciel de travail collaboratif, groupware, serveurs communs) / des logiciels de définition/suivi des processus (workflow) / des logiciels « métier» (spécifiques à votre activité) / des certificats électroniques ?

Dans votre entreprise utilise-t-on Internet pour tout ou partie des appels téléphoniques (systèmes type skype, « voix sur IP »...) ? [oui/non]

Votre entreprise possède-t-elle un service de visioconférence (webcam ou salle équipée) ? [oui/non]

Votre entreprise a-t-elle mis en place un ERP/PGI ? [oui/non]

Votre entreprise a-t-elle un site web? [oui/non] Quelles sont ses fonctionnalités? Commandes et réservations en ligne ; paiements en ligne [multi-choix possible].

\section{Dynamisme du marché}

Diriez-vous que votre marché principal est [réponse au choix] : en croissance / stable / en décroissance

\section{Secteur d'activité}

[Catégorisation réalisée sur la base des codes APE fournis par les répondants] : Industries agricole et alimentaire / Industrie des biens de consommation / Industrie des biens d'équipement et automobile / Industrie des biens intermédiaires et Energie / Construction / Commerce et réparation automobile / Commerce de gros / Commerce de détail / Transport / Activités financières et immobilières / Services aux entreprises. 